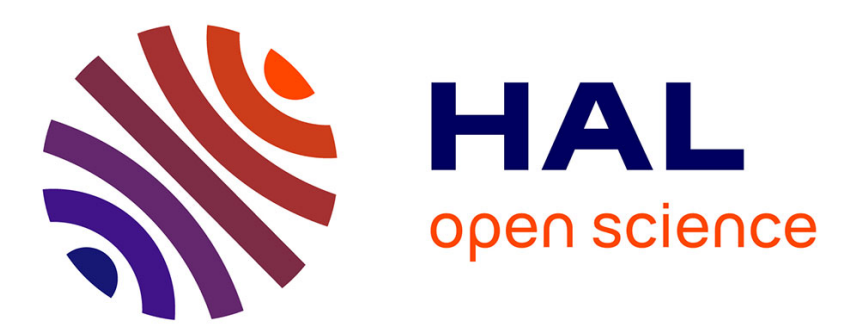

\title{
A spectral approach for sifting process in Empirical Mode Decomposition
}

\author{
Oumar O. Niang, Eric Deléchelle, Jacques Lemoine
}

\section{To cite this version:}

Oumar O. Niang, Eric Deléchelle, Jacques Lemoine. A spectral approach for sifting process in Empirical Mode Decomposition. IEEE Transactions on Signal Processing, 2010, 58 (11), pp.5612 - 5623. 10.1109/TSP.2010.2055563 . hal-00662003

\section{HAL Id: hal-00662003 https://hal.science/hal-00662003}

Submitted on 2 Feb 2012

HAL is a multi-disciplinary open access archive for the deposit and dissemination of scientific research documents, whether they are published or not. The documents may come from teaching and research institutions in France or abroad, or from public or private research centers.
L'archive ouverte pluridisciplinaire HAL, est destinée au dépôt et à la diffusion de documents scientifiques de niveau recherche, publiés ou non, émanant des établissements d'enseignement et de recherche français ou étrangers, des laboratoires publics ou privés. 


\title{
A Spectral Approach for Sifting Process in Empirical Mode Decomposition
}

\author{
Oumar Niang, Éric Deléchelle, and Jacques Lemoine
}

\begin{abstract}
In this paper, we propose an alternative to the algorithmic definition of the sifting process used in the original Huang's empirical mode decomposition (EMD) method. Although it has been proven to be particularly effective in many applications, EMD method has several drawbacks. The major problem with EMD is the lack of theoretical Framework which leads to difficulties for the characterization and evaluation this approach. On top of the mathematical model, there are other concerns with mode mixing and transient phenomena, such as intermittency or pure tones separation. This paper follows a previous published nonlinear diffusion-based filtering to solve the mean-envelope estimation in sifting process. The major improvements made in this present work are a non-iterative resolution scheme for the previously proposed partial differential equation (PDE), a new definition of the stopping function used in the PDE framework, and finally an automatic regularization process based on inverse problem theory to deal with mode mixing or transient detection problem. Obtained results confirm good properties of the new version of the PDE-based sifting process and its usefulness for decomposition of various kinds of data. The efficiency of the method is illustrated on some examples using informative and pathological signals for which standard EMD algorithm fails.
\end{abstract}

Index Terms-Diffusion equations, eigenvalues, empirical mode decomposition (EMD), inverse problems, mean-envelope, mode mixing, Tikhonov regularization, transient.

\section{INTRODUCTION}

$\mathbf{T}$ HE empirical mode decomposition (EMD) [1] is an algorithm that produces a representation of a discrete signal in terms of elementary modes, termed intrinsic mode functions (IMFs). This nonlinear decomposition method extracts, through an iterative sifting procedure, IMFs which are conditioned on the function to be represented. These IMFs are local averages which are extracted from highest local frequencies to the lowest through an amazingly effective and simple collection of algorithms. Each IMF generated is typically completely unrelated

Manuscript received November 18, 2009; accepted June 08, 2010. Date of publication June 28, 2010; date of current version October 13, 2010. The associate editor coordinating the review of this manuscript and approving it for publication was Prof. Xiang-Gen Xia.

O. Niang is with the Département Génie Informatique et Télécommunications, Ecole Polytechnique de Thiès, 64551 Dakar-Fann, Sénégal, and also with the Laboratoire Images, Signaux et Systèmes Intelligents (LISSI-E.A.3956) Université Paris Est Créteil Val-de-Marne, Créteil 94010, France and with the Laboratoire d'Analyse Numérique et d'Informatique (LANI), Universite Gaston Berger (UGB), Saint-Louis, Sénégal (e-mail: oniang@ucad.sn; niangom@yahoo.fr).

É. Deléchelle and J. Lemoine are with the Laboratoire Images, Signaux et Systèmes Intelligents (LiSSi-E.A.3956)-Université Paris Est Créteil Val-deMarne, Créteil 94010, France (e-mail: lemoinedoyen.sciences@univ-paris12. fr).

Color versions of one or more of the figures in this paper are available online at http://ieeexplore.iee.org.

Digital Object Identifier 10.1109/TSP.2010.2055563 to the ones of higher local frequency which were extracted earlier in the process. This particular representation in many cases is amenable to further analysis using the Hilbert transform.

There are many variants of the sifting procedure, many of which were suggested in [1], [2]. To date, only one variant has led to a convergence analysis, namely the B-spline method [3]. The elementary component IMF's, as defined by Huang [1], have been characterized in terms of solutions of Sturm-Liouville equations [4] but the sifting procedure and an understanding of the decomposition process have eluded researchers. Sifting is an iterative procedure whose limit determines a special local average of the signal. The analogy with wavelets would be that this is a coarsening operation to a signal with fewer extrema (and therefore lower frequency of oscillation) and that the difference between this (nonlinear) projection and the signal would correspond to a wavelet representation at that resolution level.

The origin of EMD is essentially algorithmic in nature, and hence, the method lacks of a solid theoretical framework. In a recent paper [5], we have proposed an analytical approach for sifting process based on Partial Differential Equation (PDE). The utility of the proposed method has been successfully demonstrated with the help of several synthetic signals, which demonstrate that this approach performs as well as the classical EMD algorithm. Following this work, we proposed here two major contributions. First, we give a new definition of the characteristic points of the signal to be decomposed. Second, a noniterative scheme to solve the coupled PDEs system for upper and lower envelopes estimation. Finally, we proposed a regularization approach to detect "pathological" cases. As a consequence, some of the inherent drawbacks of the original EMD algorithm are taken into account, and the decomposition obtained, from particular signal cases, show very good results.

\section{EMD BASICS}

This section presents the EMD method [1] in a nutshell. All the details regarding the implementation of EMD algorithm and Matlab EMD codes are fully available in [7]. EMD method iteratively decomposes a complex signal (i.e., a signal with several characteristic time scales coexisting) into several elementary AM-FM type components, called Intrinsic Mode Functions (IMFs).

The underlying principle of this decomposition is to locally identify the most rapid oscillations in the signal, defined as a waveform interpolating interwoven local maxima and minima. To do so, the local maxima points (and, respectively, the local minima points) are interpolated with a cubic spline, to determine the upper (and, respectively, the lower) envelope. The mean envelope (i.e., the half sum of the upper and the lower envelopes) 
is then subtracted from the initial signal, and the same interpolation scheme is reiterated on the remainder. The so-called sifting process terminates when the mean envelope is reasonably zero everywhere, and the resultant signal is designated as the first IMF. The higher order IMFs are iteratively extracted applying the same procedure for the initial signal, after removing the previous IMFs. In the original definition of IMF [1], to be an IMF a signal must satisfy two criteria, the first one being that the number of local maxima and the number of local minima must differ by at most one, and the second, the mean of its upper and lower envelopes must equal zero. In the original version of EMD algorithm, Huang et al. [1] have to determine a criterion for the sifting process to stop. This is accomplished by limiting the size of the standard deviation $S D$ computed from the two consecutive sifting results.

The first IMF $i m f_{1}$, and the first residual $r_{1}$, of a signal $s_{0}=$ $s$ are found by iterating through the (inner) loop of Algorithm 1. The sifting procedure performed on $s_{0}$ can then be performed on the residual $r_{1}$ to obtain $i m f_{2}$ and $r_{2}$ and repeated (outer loop) until the residual is reasonably not too small. So, for any onedimensional discrete signal, $s[n]$, EMD can finally be presented with the following representation:

$$
s[n]=\sum_{k=1}^{K} i m f_{k}[n]+r_{K}[n]
$$

where $i m f_{k}$ is the $k$ th IMF (or mode) of the signal, and $r_{K}$ is the final residual trend (a low order polynomial component). Sifting procedure generates a finite (and limited) number of IMFs that are nearly orthogonal to each other.

Algorithm 1 : Sifting process at level $k$ - STANDARD FORM [1]

1: set $i \leftarrow 0, h_{k, 0} \leftarrow r_{k} \quad \triangleright$ Initialization

2: repeat $\triangleright$ Loop

3: set $i \leftarrow i+1, h_{k, i} \leftarrow h_{k, i-1}$

4: find extrema of $h_{k}$ (minima and maxima)

5: compute upper envelope $e^{+}$by interpolating between maxima

6: compute lower envelope $e^{-}$by interpolating between minima

7: compute proto-local mean $l_{k, i} \leftarrow\left(e^{+}+e^{-}\right) / 2$

8: update proto-mode function $h_{k, i+1} \leftarrow h_{k, i}-l_{k, i}$

9: until $h_{k, i+1}$ is an IMF. $\quad \triangleright$ End Loop

10: set $i m f_{k+1} \leftarrow h_{k, i+1}$, and $r_{k+1} \leftarrow r_{k}-i m f_{k+1} \quad \triangleright$

Result

In all cases, an IMF can be viewed as a (nonlinear) frequency narrow-band wavelet $\varphi$ with amplitude modulation by a lower frequency signal $a[n]$
In stochastic situations involving broadband noise, one can make an interpretation of EMD in terms of a constant $-Q$ filter bank [8], [9].

\section{EMD DRAWBACKS AND IMPROVEMENTS}

The implementation of EMD requires some attention to deal with the selection of the stopping criteria for the sifting process and the management of the end points for cubic splines interpolation in the EMD process. But another important obstacles, to this approach, lie on the problem of "intermittencies" and "mode mixing." Before to present a novel formulation attempting to improve the standard EMD approach, we review some of the published works dealing with these drawbacks.

\section{A. Stopping Criterion}

When testing for IMF criteria in the sifting process, two tests must be passed. The number of extrema and zero-crossings must not differ by more than one. The second criterion is that the mean between the upper and lower envelopes must be close to zero according to some criterion. The criteria that have been considered so far in [1] leads, in certain situations, to overdecomposition. As an improvement, Rilling et al. [2] proposed an approach to choosing stopping criteria in order to guarantee globally small fluctuations in the mean while taking into account locally large excursions. This is accomplished by introducing at each sifting iteration an amplitude and an evaluation function. They used two thresholds, one designed to ensure globally small fluctuations in the mean of the cubic splines from zero, and the second allowing small regions of locally large deviations from zero.

\section{B. Border Effects}

However, although a powerful method, EMD must be used cautiously when extracting the IMFs. As mentioned in [2] when locating the extrema of the time series at each sifting process, the end points (boundary conditions) of the time series are to be treated differently in order to minimize error propagations due to finite observations in length. There are a variety of techniques that have been used in past studies on EMD, and [2] offers one of the simplest yet very robust by mirrorizing the extrema at the time series boundary conditions.

\section{Intermittencies}

As noted in [1] and [10], intermittency (or riding waves) is a major obstacle to the use of EMD on many signals. Intermittency for example occurs in turbulent flow or in any signal that is constantly changing such as speech. In this case we refer to intermittency as a component at a particular time scale either coming into existence or disappearing from a signal entirely. Since EMD locally pulls out the highest frequency component as the current IMF, intermittency in a signal means that the frequency tracked by a particular IMF will jump as the intermittent component begins or ends. This situation is illustrated with signal in Fig. 1(a) where we can see why transient signals or intermittency artifacts must be taking out in the beginning of an EMD sifting process.

A solution to intermittency is proposed in [11] in which a change in the choice of extrema for the envelopes limits the

$$
i m f_{k}[n]=a_{k}[n] \varphi_{k}[n] .
$$




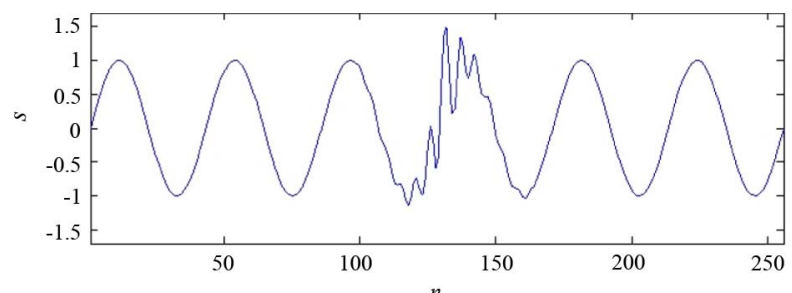

(a)

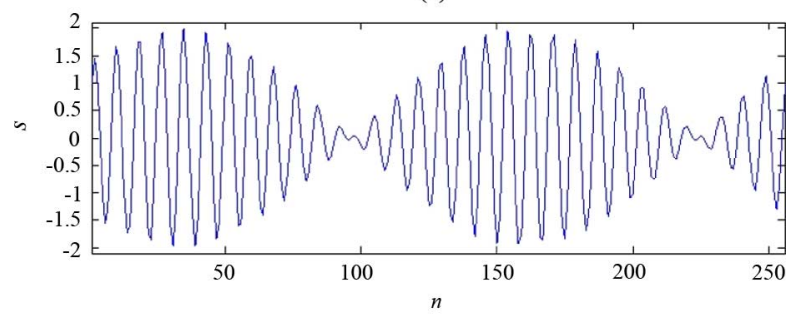

(b)

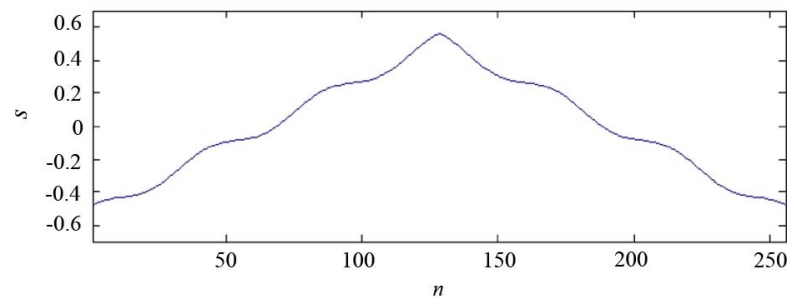

(c)

Fig. 1. Some pathological cases for original EMD approach. (a) A mixed mode signal showing an intermittency around $n \approx 140$. (b) A tow-tones signal. (c) A wave-like signal with only one extrema at $n=128$.

scale over which the sifting process allows a component to pass. Intermittent affect the outcome of the IMF generation. In general, riding waves are defined as a transient signals that are interrupting the predominant pattern of the wave. Particular to intermittency detection, intermittency is defined as any set of a maximum and minimum extrema such that the distance between the adjacent extrema points is less than the designated threshold, $\nu$. In original EMD during the process of removing intermittency from an IMF, a search is made in the signal for all maximum and minimum extrema which the distance between any two extrema is greater than or equal to $\nu$. In order to separate the intermittency artifacts into a different IMF, those points found during the process are removed, and all values that are less than the threshold are retained, and the EMD calculation is continued. There is currently no automatic heuristics for selection of threshold values, due to the complexity of such analysis. A recent method to alleviate some of the common problems of EMD such as mode mixing is Ensemble EMD (EEMD) introduced by Wu and Huang [12] to try increasing the robustness of EMD. In EEMD, the final EMD is estimated by averaging numerous EMD runs with the addition of noise.

Another solution involving a masking signal is presented in [13]. The masking signal technique allows EMD to be used to separate intermittency from other components that would be inseparable with standard EMD algorithm. This approach is based on an elegant usage of the standard Algorithm 1. The basic idea is to insert a masking signal, a single sine tone for which frequency is chosen appropriately, that prevents lower frequency components from being included in the first IMF, thus capturing intermittency components. Because of its focus on frequency, the corresponding implementation is presented in Algorithm 2. Hence, we see that intermittency management is performed through a double sifting process and assuming the knowledge of the 'cut-off' frequency $\omega_{m}$ imposed by the masking signal (see [13] for more details on frequency selection).

Algorithm 2 : Sifting process at level $k$ - MASKING SIGNAL [13]

1: choose masking tone $m$ at frequency $\omega_{m} \quad \triangleright$ Initialization

2: set $r_{k}^{+} \leftarrow r_{k}+m \quad \triangleright 1$ st Proc.

3: perform Algorithm 1 on $r_{k}^{+}$to obtain $i m f_{k+1}^{+}$

4: set $r_{k}^{-} \leftarrow r_{k}-m \quad \triangleright$ 2nd Proc.

5: perform Algorithm 1 on $r_{k}^{-}$to obtain $i m f_{k+1}^{-}$

6: set $i m f_{k+1} \leftarrow\left(i m f_{k+1}^{+}+i m f_{k+1}^{-}\right) / 2$, and

$r_{k+1} \leftarrow r_{k}-i m f_{k+1} \quad \triangleright$ Result

\section{Pure Tones Separation}

When considering the performance of EMD on a combination of pure tones, it is important to note that a sum of pure tones can be rewritten as a product of two tones.

$$
\begin{aligned}
s(t) & =\cos 2 \pi f_{1} t+\cos 2 \pi f_{2} t \\
& =\cos 2 \pi\left(f_{1}-f_{2}\right) t \cos 2 \pi\left(f_{1}+f_{2}\right) t .
\end{aligned}
$$

If $f_{1} \approx f_{2}$, then we observe that the resulting signal can be viewed as Amplitude Modulated (AM) wave, see Fig. 1(b). The performance of EMD on pairs of pure tones is discussed in [2], where the authors show that we can define a confusion frequency band $B\left(f_{1}\right)=\left[\alpha_{\rho} f_{1}, f_{1}\right]$, with $\alpha_{\rho}<1$, such that $f_{2}<f_{1}$ and $f_{2} \in B\left(f_{1}\right)$ cannot be separated.

The previous cited approach, Algorithm 2 [13], using masking signal for intermittency management performs as well for tones separation. Hence, the masking signal technique allows EMD to be used to separate components that are similar in frequency that would be inseparable with standard EMD algorithm.

\section{E. Variation With Non Local Extrema}

In its actual version, EMD acts on oscillating signals that present local extrema. Consider the simple case for which a signal $s(t)$ is defined as

$$
s(t)=\alpha t+\sin \omega_{0} t, \quad a \leq t \leq b
$$

with $\omega_{0}<|\alpha|$. So, the temporal derivative of $s(t)$ is

$$
\dot{s}(t)=\alpha+\omega_{0} \cos \omega_{0} t
$$

Hence $\dot{s}(t) \neq 0$ on interval $[a, b]$, and no minima and maxima are defined. See Fig. 1(c) for an example of such a wavelike signal. 
Hence, the two components would be inseparable with standard EMD Algorithm 1.

\section{PDE-BASED EMD}

As proposed in [5], a possible form for fourth order diffusion equation is

$$
\frac{\partial s(x, t)}{\partial t}+\frac{\partial}{\partial x}\left(g(x, t) \frac{\partial^{3} s(x, t)}{\partial x^{3}}\right)=0
$$

where $g(x, t)$ is the stopping function possibly depending on both position and time, and where the time variable is artificial, and measures the degree of processing (e.g., smoothing) of the signal, as opposed to a real time. Equation (2) can be viewed as a long-range diffusion (LRD) equation (see, for example, $[14$, p. 244]), with thresholding function $g(x)$ depending only on position (constant in time) and more precisely on some characteristic fix points of the signal to decompose. After derivation, (2) reads

$$
s_{t}(x, t)+\delta_{x}^{1}\left(g(x) \delta_{x}^{3} s(x, t)\right)=0
$$

where the subscript $t$ denotes partial differentiation with respect to the variable $t$ and $\partial_{x}^{q}$ denotes partial differentiation of order $q$ with respect to the variable $x$. In the following, we use the notation $s_{0}(x)=s(x, t=0)$ for initial condition and $s_{\infty}(x)=s(x, t=\infty)$ for asymptotic solution of (3) whose existence is proven in [6].

In order to implement sifting procedure in a PDE-based framework, the following processes are based on the definition of characteristic points of a function: (i) turning-points; (ii) curvature-points. Thanks to these characteristic points, we define a coupled PDEs system in sifting process to estimate lower and upper envelopes.

\section{A. A Coupled PDEs System}

In a previous paper [5] and more in [6], we have introduced and studied a coupled PDEs system where the stopping functions $g^{ \pm}$, for upper and lower envelopes, are related to maxima and minima points (MMP), so $g^{ \pm}$depend on both first and second derivatives of $s_{0}$, in such a way such that $g^{+}=0$ at maxima of $s_{0}$, in the same way $g^{-}=0$ at minima of $s_{0}$. So, LRD acts only between two consecutive maxima (respectively, minima) points until fourth-order derivative of $s(x, t)$ is canceled. Since, stopping functions are piecewise constant, after convergence of the PDE solution (see [6]) the resulting asymptotic signal $s_{\infty}^{+}(x)=s_{\infty}^{+}(x, t=\infty)$ (respectively, $\left.s_{\infty}^{-}(x)=s_{\infty}^{-}(x, t=\infty)\right)$ is a piecewise cubic polynomial curve interpolating the successive maxima (respectively, minima) of signal.

Then, the coupled PDEs system, based on (3) reads

$$
\left\{\begin{array}{l}
s_{t}^{+}(x, t)=-\delta_{x}^{1}\left(g^{+}(x) \delta_{x}^{3} s^{+}(x, t)\right) \\
s_{t}^{-}(x, t)=-\delta_{x}^{1}\left(g^{-}(x) \delta_{x}^{3} s^{-}(x, t)\right)
\end{array}\right.
$$

with $s^{+}(x, t=0)=s^{-}(x, t=0)=s_{0}$, and where stopping functions, $g^{ \pm}$, depend on signal derivatives, with $0 \leq g^{ \pm} \leq 1$. In [5], a reasonable choice for stopping functions was

$$
g^{ \pm}(x)=\frac{1}{9}\left[\left|\operatorname{sgn}\left(\delta_{x}^{1} s_{0}(x)\right)\right| \pm \operatorname{sgn}\left(\delta_{x}^{2} s_{0}(x)\right)+1\right]^{2} .
$$

After convergence of system (4) asymptotic solutions $s_{\infty}^{+}(x)$ and $s_{\infty}^{-}(x)$, stand, respectively, for upper and lower envelopes of signal $s_{0}$. Hence, mean-envelope of $s_{0}$ is obtained by

$$
s_{\infty}(x)=\frac{1}{2}\left[s_{\infty}^{+}(x)+s_{\infty}^{-}(x)\right] .
$$

In the case of signal variation with nonlocal extrema, (5) for stopping functions is not adapted. As the algorithmic version of the EMD, such a signal is not decomposed into IMFs with the PDE version. In order to discard this drawback, we proposed here a another possible formulation for stopping functions based on characteristic points defined by maximum curvature points (MCP) of $s_{0}$. So, the new formulation reads

$$
g^{ \pm}(x)=\frac{1}{9}\left[\left|\operatorname{sgn}\left(\delta_{x}^{3} s_{0}(x)\right)\right| \pm \operatorname{sgn}\left(\delta_{x}^{2} s_{0}(x)\right)+1\right]^{2}
$$

where $\delta_{x}^{3}$ is used in place of $\delta_{x}^{1}$ in (5), so that stopping function $g^{+}$(respectively, $g^{-}$) is zeroed at local negative (respectively, positive) curvature maxima of $s_{0}$.

\section{B. A More General PDE-Interpolator With Tension Parameter}

A possible more general form for (3) is

$$
s_{t}^{ \pm}=\delta_{x}^{1}\left(g^{ \pm}\left(\alpha \delta_{x}^{1} s^{ \pm}-(1-\alpha) \delta_{x}^{3} s^{ \pm}\right)\right)
$$

adding Laplacian term to biharmonic one. So, in this form $\alpha$ is the tension parameter, and ranges from 0 to 1 . Zero tension $\alpha=0$ leads to the biharmonic equation for (3) and corresponds to the minimum curvature construction for upper and lower envelopes. The case $\alpha=1$ corresponds to infinite tension, resulting on piecewise linear envelopes.

\section{Numerical Resolutions}

Numerical resolutions for coupled PDEs system based on (7) is implemented in [5] via classical iterative Crank-Nicolson or Du Fort and Frankel schemes.

Equation (7) can be resolved numerically in its discrete explicit version

$$
\mathbf{S}^{k+1}=\mathbf{S}^{k}+\Delta t \mathbf{A} \mathbf{S}^{k}, \quad \mathbf{S}^{0}=\mathbf{S}_{0}
$$

where $\mathbf{S}=(s[1], \ldots, s[N])^{\mathrm{T}}$ is the column vector of signal samples for upper or lower envelopes $s^{+}$or $s^{-}$and $\mathbf{A}$ is a matrix formed with finite difference approximation coefficients of second- and fourth-order differential operators, as

$$
\mathbf{A}=\mathbf{D}_{1}\left(\mathbf{G}\left(\alpha \mathbf{D}_{1}-(1-\alpha) \mathbf{D}_{3}\right)\right)
$$

and $\mathbf{D}_{i}$ the finite difference operator of the $i^{\text {th }}$ derivative. So the explicit form is

$$
\mathbf{S}^{k+1}=(\mathbf{I}+\Delta t \mathbf{A}) \mathbf{S}^{k}, \mathbf{S}^{0}=\mathbf{S}_{0}
$$

with $\mathbf{I}$ the identity matrix. This scheme is known to be stable conditionally to time step $\Delta t$. An unconditionally stable scheme based on an implicit version reads

$$
\mathbf{S}^{k+1}=\mathbf{S}^{k}+\Delta t \mathbf{A} \mathbf{S}^{k+1}, \quad \mathbf{S}^{0}=\mathbf{S}_{0}
$$



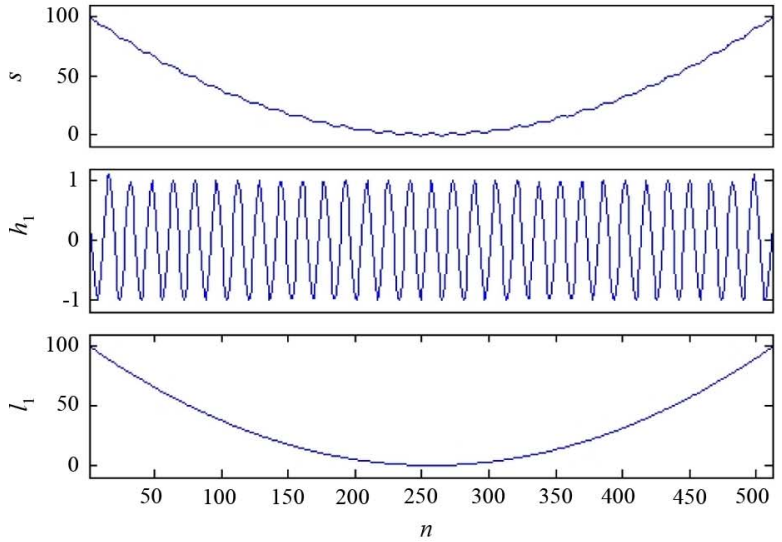

(a)

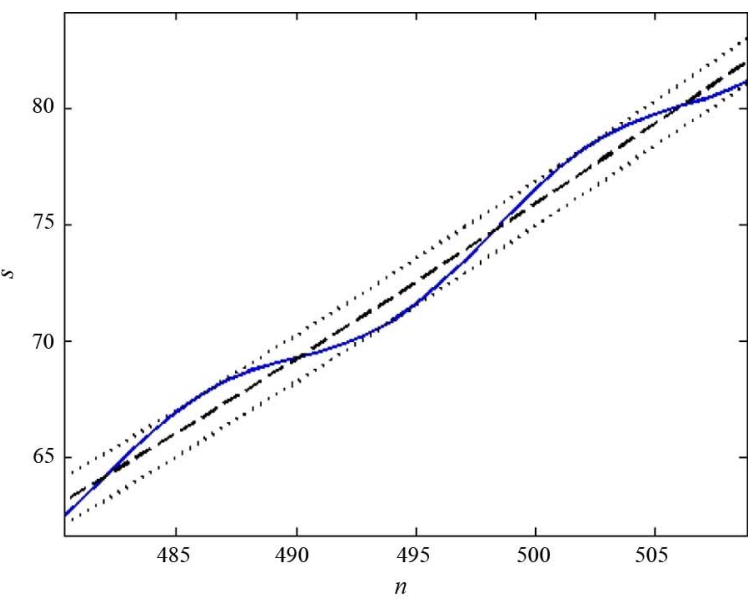

(b)

Fig. 2. An example of signal decomposition with nonlocal extrema. (a) Top plot, original signal with its upper, lower, and mean envelopes. Middle plot, proto-mode signal $h_{1}(t)$ after one iteration of sifting process. Bottom plot, proto-local mean envelope $l_{1}(t)$. (b) A detail on signal and its lower, upper, and mean envelopes.

leading to the following numerical resolution:

$$
\mathbf{S}^{k+1}=(\mathbf{I}-\Delta t \mathbf{A})^{-1} \mathbf{S}^{k}, \quad \mathbf{S}^{0}=\mathbf{S}_{0} .
$$

Both in (8) and (9) matrix $\mathbf{G}$ is construct with discrete version of stopping function values $g(x)$. The discrete version of $g$ for (5) and (6) are done by

$$
\begin{aligned}
& g\left(\delta_{x}^{1} s_{0}, \delta_{x}^{2} s_{0}\right)=g\left(D_{1} s_{0}, D_{2} s_{0}\right) \\
& g\left(\delta_{x}^{3} s_{0}, \delta_{x}^{2} s_{0}\right)=g\left(D_{1} D_{2} s_{0}, D_{2} s_{0}\right)
\end{aligned}
$$

where $D_{2} z=D^{+} D^{-} z$, and $D_{1} z=\mathrm{m}\left(D^{+} z, D^{-} z\right)$ with $D^{+}$and $D^{-}$forward and backward first difference operators on the $x$ dimension, and where $\mathrm{m}(a, b)$ stands for the minmod limiter $\mathrm{m}(a, b)=0.5(\operatorname{sgn} a+\operatorname{sgn} b) \min (|a|,|b|)$.

Some results of signal decompositions obtained with (5) and implicit scheme (9) are reported in [5].

\section{SPECTRAL APPROACH}

In this section, we introduced a spectral approach for the resolution of (7) with a noniterative scheme.

\section{A. Linear System Equations Resolution}

The approach is based on the fact that (7) can be decomposed into a linear system of equations of the following two forms:

$$
\mathbf{S}^{k+1}=\mathbf{L} \mathbf{S}^{k}, \quad \mathbf{S}^{0}=\mathbf{S}_{0}, \quad k \geq 0
$$

from explicit numerical scheme (8), or alternatively from implicit numerical scheme by (9)

$$
\mathbf{S}^{k+1}=\hat{\mathbf{L}}^{-1} \mathbf{S}^{k}, \quad \mathbf{S}^{0}=\mathbf{S}_{0}, \quad k \geq 0
$$

where $\mathbf{L}$ and $\hat{\mathbf{L}}$ are the linear operators including stopping function values and differential operator formed by fourth order and second order. So, referring to numerical schemes (8) and (9), L and $\hat{\mathbf{L}}$ are given by

$$
\begin{aligned}
& \mathbf{L}=\mathbf{I}+\Delta t \mathbf{A} \\
& \hat{\mathbf{L}}=\mathbf{I}-\Delta t \mathbf{A} .
\end{aligned}
$$

As implicit scheme is unconditionally stable for all step size $\Delta t$, we will concentrate on this scheme for the rest of the document.

\section{B. Asymptotic Solution}

In our case, we are looking for the asymptotic solution of (7). So, noting that the iterative scheme(10) can be rewrite in term of initial solution $\mathbf{S}_{0}$ as

$$
\mathbf{S}^{k}=\left(\hat{\mathbf{L}}^{-1}\right)^{k} \mathbf{S}_{0}, \quad k \geq 1
$$

after convergence, the asymptotic solution $\mathbf{S}_{\infty}$ is given by

$$
\mathbf{S}_{\infty}=\left(\hat{\mathbf{L}}^{-1}\right)^{\infty} \mathbf{S}_{0} .
$$

The operator matrix $\hat{\mathbf{L}}$, as a real-valued has real-valued eigenvalues. More, it easy to show that eigenvalues of $\hat{\mathbf{L}}$ are always greater or equal to 1 . Then, eigenvalues $\lambda_{n}$ of $\hat{\mathbf{L}}^{-1}$ are always smaller or equal to $1,\left(0<\lambda_{n} \leq 1\right)$. Finding the eigenvalues and eigenvectors of the operator matrix is closely related to its decomposition

$$
\hat{\mathbf{L}}^{-1}=\mathbf{V} \mathbf{\Lambda} \mathbf{V}^{-1}
$$

where $\mathbf{V}$ is a matrix of $\hat{\mathbf{L}}^{-1}$, s eigenvectors $\mathbf{v}_{n}$ and $\Lambda$ is a diagonal matrix having $\hat{\mathbf{L}}^{-1}$ 's eigenvalues $\lambda_{n}$ at the diagonal. It is easy to see that we have

$$
\left(\hat{\mathbf{L}}^{-1}\right)^{k}=\left(\mathbf{V} \boldsymbol{\Lambda} \mathbf{V}^{-1}\right)^{k}=\mathbf{V} \Lambda^{k} \mathbf{V}^{-1} .
$$

So, the asymptotic solution of (13) is obtained by

$$
\mathbf{S}_{\infty}=\left(\mathbf{V} \boldsymbol{\Lambda}^{\infty} \mathbf{V}^{-1}\right) \mathbf{S}_{0} .
$$

As $\Lambda$ is a diagonal matrix with eigenvalues $\lambda_{n}=1$ only at loci where matrix $\mathbf{G}$ is zeroed, i.e., where stopping function $g[n]=0$, and $0<\lambda_{n}<1$ where $g[n]>0$, then, the asymptotic eigenvalue matrix $\Lambda^{\infty}$ is a diagonal matrix with eigenvalues $\lambda_{n}^{\infty}=1$ only at loci where matrix $\mathbf{G}$ is zeroed, and $\lambda_{n}^{\infty}=0$ where $g[n]>0$. 
So, we can define the asymptotic eigenvalue matrix $\boldsymbol{\Lambda}^{\infty}=\operatorname{diag}\left(\lambda_{1}^{\infty}, \ldots, \lambda_{N}^{\infty}\right)$ in the following manner:

$$
\lambda_{n}^{\infty}=\left\{\begin{array}{ll}
1, & \lambda_{n}=1 \\
0, & \lambda_{n}<1
\end{array}, n=1, \ldots, N .\right.
$$

\section{Quasi-Asymptotic Solution}

In order to solve intermittency problem [1], [11], we propose a solution to taking out transient signal during sifting process.

We define the quasi-asymptotic eigenvalue matrix $\Lambda_{\epsilon}^{\infty}=\operatorname{diag}\left(\lambda_{\epsilon 1}^{\infty}, \ldots, \lambda_{\epsilon N}^{\infty}\right)$ as depicted

$$
\lambda_{\epsilon n}^{\infty}=\left\{\begin{array}{ll}
1, & 1-\epsilon \leq \lambda_{n} \leq 1 \\
0, & \lambda_{n}<1-\epsilon
\end{array}, n=1, \ldots, N\right.
$$

where $0 \leq \epsilon \leq 1$ is a threshold so that $\boldsymbol{\Lambda}_{0}^{\infty}=\boldsymbol{\Lambda}^{\infty}, \boldsymbol{\Lambda}_{1}^{\infty}=\mathbf{I}$, and the quasi-asymptotic solution is now

$$
\mathbf{S}_{\infty}= \begin{cases}\left(\mathbf{V} \Lambda^{\infty} \mathbf{V}^{-1}\right) \mathbf{S}_{0}, & \epsilon=0 \\ \left(\mathbf{V} \Lambda_{\epsilon}^{\infty} \mathbf{V}^{-1}\right) \mathbf{S}_{0}, & 0<\epsilon<1 \\ \mathbf{S}_{0}, & \epsilon=1 .\end{cases}
$$

So, during the sifting process in EMD, one can impose an intermittency frequency $\omega_{\epsilon}$ so that the resulting IMF will not contains any frequency components smaller than $\omega_{\epsilon}$. Now, the question is "how to choose $\epsilon$ ?" We propose, in Section VII, a possible solution to this question.

\section{IMPLEMENTATION AND RESULTS}

\section{A. Implementation}

The spectral resolution for sifting process is resumed in Algorithm 3.

Algorithm 3 : Sifting process at level $k$ - SPECTRAL APPROACH

1: set $i \leftarrow 0, h_{k, 0} \leftarrow r_{k}$, choose $\epsilon \quad \triangleright$ Initialization

2: repeat $\triangleright$ Loop

3: set $i \leftarrow i+1, h_{k, i} \leftarrow h_{k, i-1}$

4: compute $g^{ \pm}$from $h_{k, i}$, using MMP (5) or MCP (6)

5: compute matrix operator $\hat{L}^{-1}(11 \mathrm{~b})$

6: perform eigendecomposition of $\hat{L}^{-1}$ (13)

7: compute asymptotic eigenvalue matrix $\Lambda_{\epsilon}^{\infty}(15)$

8: find upper and lower envelopes $e^{+}$and $e^{-}(16)$

9: compute proto-local mean $l_{k, i} \leftarrow\left(e^{+}+e^{-}\right) / 2$

10: update proto-mode function $h_{k, i} \leftarrow h_{k, i}-l_{k, i}$

11: untill $h_{k, i+1}$ is an IMF $\triangleright$ End Loop

12: set $i m f_{k+1} \leftarrow h_{k, i+1}$, and $r_{k+1} \leftarrow r_{k}-i m f_{k+1} \quad \triangleright$ Result

\section{B. Some Results}

1) Example 1 (Locally Oscillating Monotone Signal): We illustrate in Fig. 2, the use of MCP-based approach in order to extract IMF from a locally oscillating but monotone signal (i.e.,
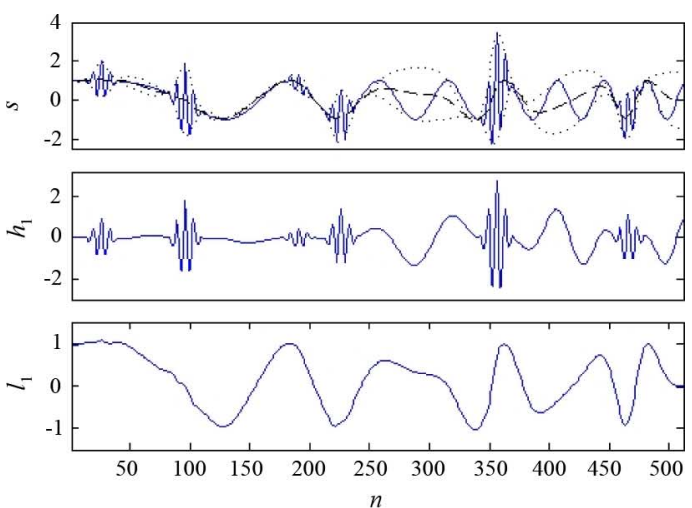

(a)
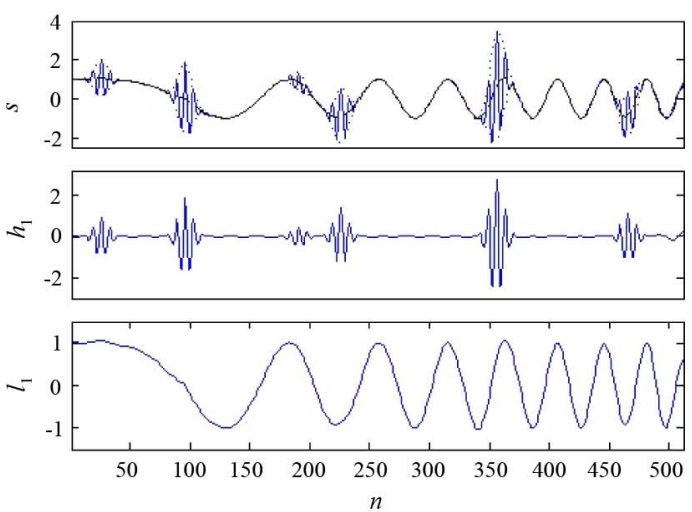

(b)

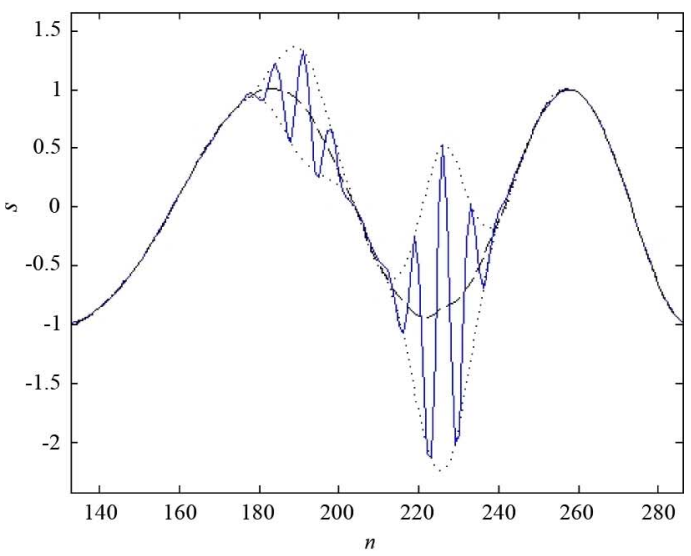

(c)

Fig. 3. An example of signal decomposition with intermittencies. (a) Eigenvalue solution with $\epsilon=0$. (b) Eigenvalue solution with $\epsilon=0.03$. (a)-(b) Top plot, original signal with upper, lower, and mean envelopes. Middle plot, protomode signal $h_{1}(t)$ after the first iteration of the sifting process. Bottom plot, the resulting proto-mean envelope signal $l_{1}(t)$. (c) A detail on signal with its envelopes.

without extrema on an interval). The composed signal is a sum of a low-amplitude pure tone $s_{1}$ with a quadratic function $s_{2}$. Results obtained after the first iteration $\left(h_{1}\right.$ and $\left.l_{1}\right)$ of the sifting process are illustrated on Fig. 2(a), where we can see that despite an absence of extrema, for example in time interval [480, 510], see Fig. 2(b), upper and lower envelopes are well detected. The classical EMD algorithm Algorithm 1 or the MMP-based approach, in Algorithm 3, will fall in this situation.

2) Example 2 (Extraction of Transient Signals): We illustrate in Fig. 3 the use of a threshold $\epsilon$ in (16) to defined values of the quasi-asymptotic eigenvalue matrix. The composed signal 

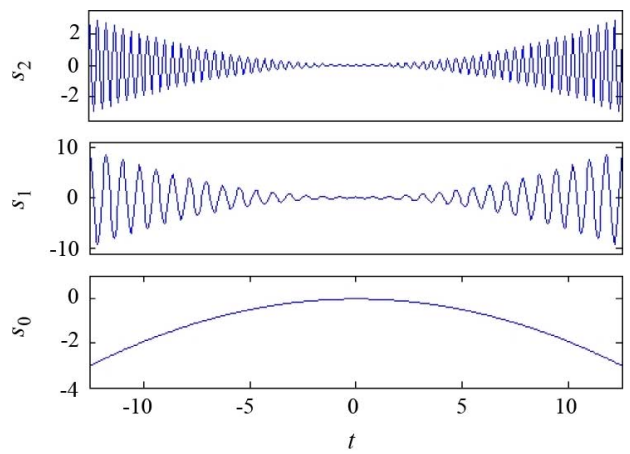

(a)
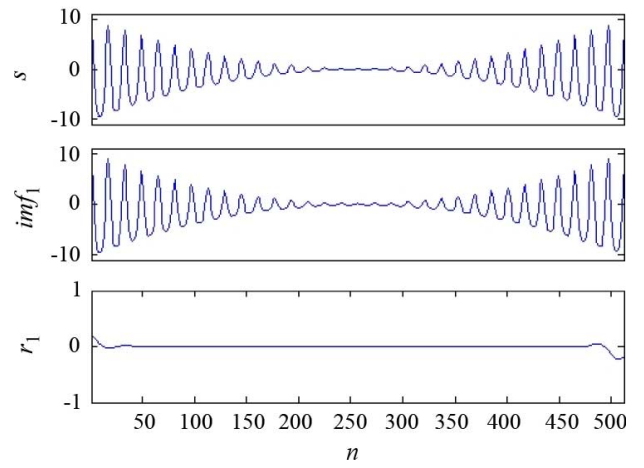

(b)

Fig. 4. AM-FM signal analysis with the standard EMD algorithm. (a) The three firsts analytical AM-modulated Fourier components issued from (18). (b) Decomposition obtained with the standard EMD approach resulting on only one IMF imf ${ }_{1}$, and a negligible residual $r_{1}$.

is a sum of a pure tone with some transient signals at different scales. Using asymptotic solution $(\epsilon=0)$, the decomposition give output where the IMF is composed on a mixing between tone oscillations and intermittencies. See Fig. 3(a) for the outputs (noted $h_{1}$ and $l_{1}$ ) after one iteration of the sifting process. We can see, Fig. 3(b), that for $\epsilon=0.03$, "manually" choosen, the proposed approach is able to extract precisely all intermittencies with a good precision [see Fig. 3(c) for a detail]. The decomposition can be interpreted as a locally adapted filtering process. See Section VII for an automatic determination of the optimal value of $\epsilon$.

3) Example 3 (Amplitude-Frequency Modulated Signal): In this example, we show how the proposed approach is able to decompose a multicomponent AM-FM signal. For this, we take the informative example of signal used in [4, example 4.4] which can be considered as a true IMF (in the sense of Huang et al.). The considered signal, with $\omega_{0}=4$, is

$$
s(t)=\frac{t^{2}+2}{16} \cos \left(\pi \sin \omega_{0} t\right), \quad-4 \pi \leq t \leq 4 \pi .
$$

Noting that, using Fourier series, $s(t)$ can be expressed as

$$
s(t)=\frac{t^{2}+2}{16}\left[J_{0}(\pi)+2 \sum_{n=1}^{\infty} J_{2 n}(\pi) \cos 2 n \omega_{0} t\right]
$$

where $J_{p}(z)$ is the Bessel function of the first kind of order $p$, and with $J_{0}(\pi)=-0.304, J_{2}(\pi)=0.485, J_{4}(\pi)=0.151$, $J_{6}(\pi)=0.014$, and $J_{2 n}(\pi)<10^{-3}$ for $n \geq 4$. The three first components of the Fourier series are illustrated on Fig. 4(a).
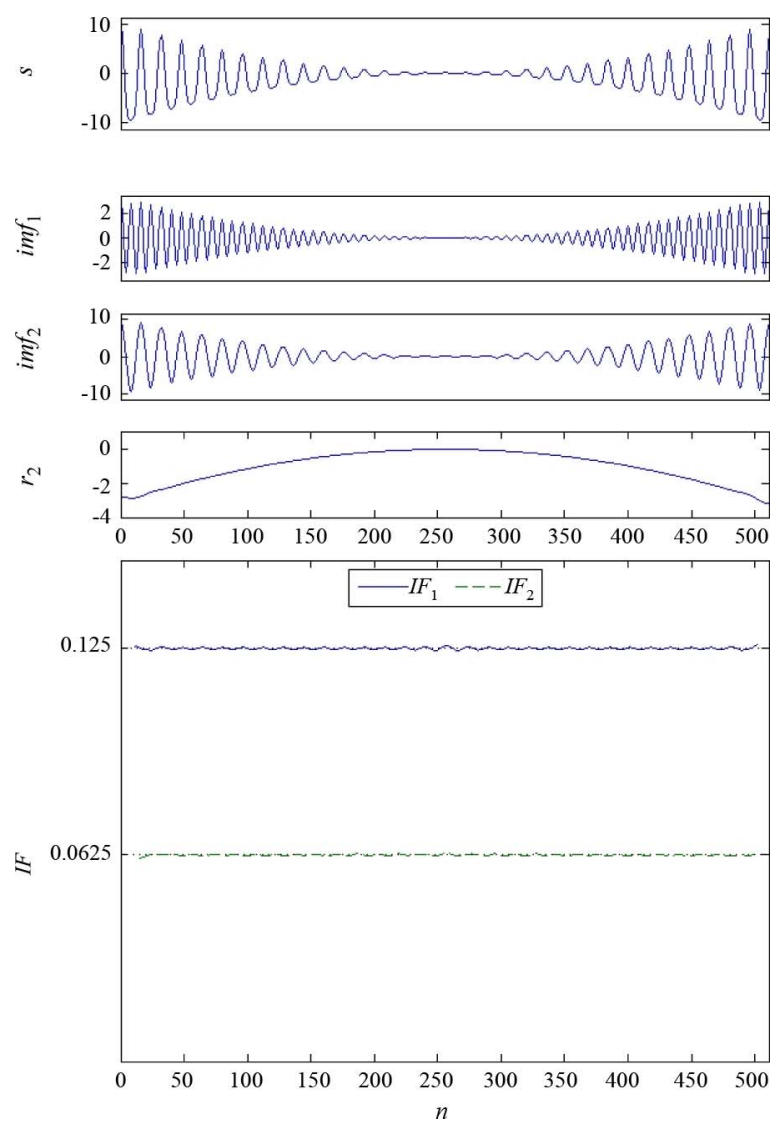

Fig. 5. AM-FM signal analysis with MCP-based approach. Top plot, signal to decomposed. Middle plots, the two IMFs and the residual. Bottom plot, instantaneous frequency representation for $i m f_{1}$ and $i m f_{2}$. The two IMFs and the residual can be compared with analytical Fourier series components given by (18) and represented on Fig. 4(a).

We sample $s(t)$ uniformly with increment $\Delta=\pi / 64$ on interval $[-4 \pi, 4 \pi-\Delta]$, resulting on a vector length of 512 points. Notice that $\left(t^{2}+2\right) / 16$ may be regarded as the Amplitude Modulated part of $s(t)$, and $\cos \left(\pi \sin \omega_{0} t\right)$ as the frequency modulated component. Hence, in classical EMD algorithm, the decomposition result shows that $s(t)$ is an IMF.

With the proposed method, we have the choice between the two definitions of the stopping function, from (5) based on minimal and maximal points (MMP) of $s(t)$ or from (6) based on maximal curvature points (MCP) of $s(t)$. The MMP-based approach give similar result that the classical EMD algorithm, i.e., $s(t)$ is considered as an IMF, resulting on a negligible residual, see Fig. 4(b). But, using the MCP-based approach, the decomposition result shows two IMFs plus a quadratic residual, see Fig. 5. Each IMF is an Amplitude Modulated sinusoidal function for which instantaneous frequency (IF), estimated from Hilbert transform [1], is illustrated in Fig. 5.

This result is consistent with the following approximation of (17), neglecting terms of rank $n>2$, we can rewrite $s(t)$ as

$$
\begin{aligned}
s(t) \approx \frac{t^{2}+2}{8} & \\
\times & \times\left[\frac{1}{2} J_{0}(\pi)+J_{2}(\pi) \cos 2 \omega_{0} t+J_{4}(\pi) \cos 4 \omega_{0} t\right] .
\end{aligned}
$$

We can see a very good agreement between $i m f_{1}$ and $s_{3}, i m f_{2}$ and $s_{1}$, and finally $r_{2}$ and $s_{0}$, Figs. 4(a) and 5 . 

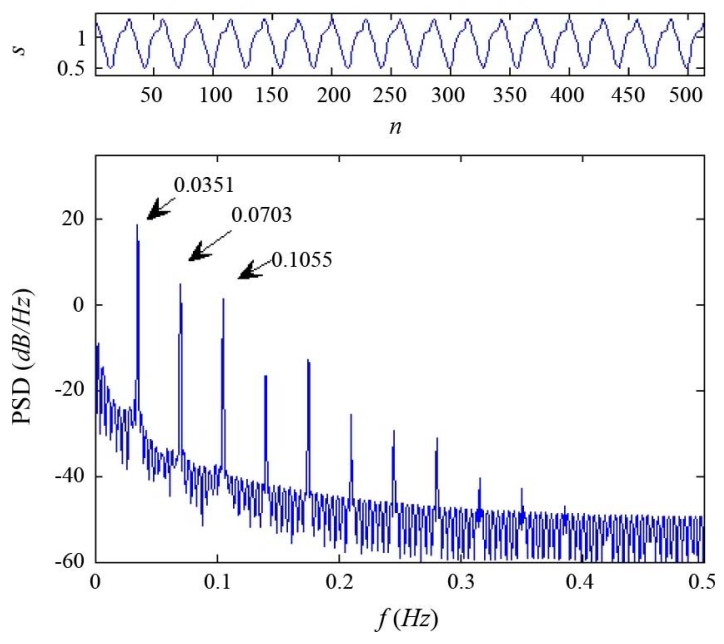

Fig. 6. An example of Mackey-Glass time series obtained from (18). To plot, the discrete MG time series. Bottom plot, power spectral density estimate via Welch's method in $\mathrm{dB}$ per unit frequency.

As described in [4], this example use a well-behaved function $s(t)$ for which the instantaneous frequency computed using Hilbert transform changes sign. So, despite the fact that $s(t)$ is viewed as an IMF with the classical EMD algorithm or with the MMP-based approach, this IMF not admits a well-behaved Hilbert transform, its Instantaneous Phase is nonmonotone and physically unrealistic. But, using the MCP-based approach, $s(t)$ is decomposed into two IMFs for which IPs are realistic and correspond to theoretical result.

4) Example 4 (Chaotic Process): In this example, we use a synthesized time series that is generated by the following Mackey-Glass (MG) time-delay differential equation [15]

$$
\dot{s}(t)=a \frac{s(t-\tau)}{1+s^{c}(t-\tau)}-b s(t) .
$$

To obtain the time series value (of 512 samples length) at integer points, we applied the fourth-order Runge-Kutta method to find the numerical solution to the above MG equation with the discrete version

$$
s[n+1]=s[n]+\Delta t\left(a \frac{s[n-\delta]}{1+s^{c}[n-\delta]}-b s[n]\right)
$$

with $a=0.25, b=0.11, c=15, \Delta t=1, \delta=10$, and initial value $s[n]=0.1$ for $n \leq 0$. The MG process and its power spectral density (PSD) are both illustrated in Fig. 6.

A first decomposition using original EMD algorithm is illustrated on Fig. 7(a). As we can see, the time series $s[n]$ is in this case essentially viewed as a FM-modulated IMF, with central frequency $C F_{1} \approx 0.035$.

With the MCP-based EMD, represented on Fig. 7(b), the result shows that $s[n]$ is decomposed on three FM-modulated IMFs. For each IMF, $i m f_{k}$, the Central Frequency $C F_{k}$ estimated from instantaneous frequency $I F_{k}$ is $C F_{k} \approx k f_{0}$, with $f 0 \approx 0.035$.

\section{Automatic Mode Mixing and Transient Detections}

In this section we propose a method to estimate, automatically, the optimal quasi-asymptotic parameter $\epsilon$, Section V-C, to deal with the problem of mode mixing and transient detections in signal as in Example 2 of Section VI-B, illustrated in Fig. 3.

\section{A. Tikhonov Regularization}

Consider the linear problem

$$
\mathbf{d}=\mathbf{A f}+\mathbf{n}
$$

where $\mathbf{f}$ the input we wish to reconstruct, $\mathbf{d}$ is the output we measure, $\mathbf{A}$ is a linear transformation, and $\mathbf{n}$ represents an additive noise process which prevents us from knowing the noise-free data $\mathbf{y}=$ Af.

Tikhonov regularization provides a framework for stabilizing the solution of possible ill-conditioned linear equations [16]. The solution of (19) using Tikhonov regularization can be written as

$$
\hat{\mathbf{f}}_{\lambda}=\underset{\mathbf{f}}{\arg \min }\left\{\|\mathbf{A f}-\mathbf{d}\|^{2}+\lambda^{2}\left\|\mathbf{L}_{p}\left(\mathbf{f}-\mathbf{f}^{\infty}\right)\right\|^{2}\right\} .
$$

This is a whole family of solutions parameterized by the weighting factor $\lambda^{2}$. Here $\lambda$ is the regularization parameter, $\mathbf{L}_{p}$ is a positive semidefinite linear transformation, $\mathbf{f}^{\infty}$ denotes the prior information about the solution $\mathbf{f}$. Typically, $\mathbf{L}_{p}$ is the identity matrix or a banded matrix approximation to the $p$ th derivative.

If the regularization parameter is very large, the effect of the model error term, $C(\mathbf{f})=\|\mathbf{A f}-\mathbf{d}\|^{2}$, is negligible to that of the prior error $\Omega(\mathbf{f})=\left\|\mathbf{L}_{p}\left(\mathbf{f}-\mathbf{f}^{\infty}\right)\right\|^{2}$ and we find that $\lim _{\lambda \rightarrow \infty} \hat{\mathbf{f}}_{\lambda}=\mathbf{f}^{\infty}$. On the other hand, if $\lambda$ is small, the problem reduces to the least-squares case considered in (19) with an extreme sensitivity to noise $\mathbf{n}$ on the data $\mathbf{d}$. So, the regularizing parameter $\lambda$ can be thought of as controlling the balance between minimizing the regularizing term $\Omega(\mathbf{f})$ and minimizing the term $C(\mathbf{f})$, which corresponds to fitting data.

\section{B. Application to Sifting Process}

In order to apply this approach to the problem of mode mixing and transient detection, we propose to take advantage of the reconstruction relation at level $k$ of the decomposition

$$
l_{k, i-1}=h_{k, i}+l_{k, i}
$$

where $h_{k, i}$ and $l_{k, i}$ are, respectively, the proto-mode and the proto-residual obtained at iteration $i$ during the sifting process, with $l_{k, 0}=r_{k}$ and $r_{0}=s$, where $s$ denotes the decomposed signal as in (1). If we make the assumption that, initially, $r_{k}$ contains mixed modes or transients, then we can identify the reconstruction relation (21) with the linear problem (19), and write after one iteration of the sifting process (e.g., $i=1$ )

$$
\mathbf{d}=r_{k}, \quad \mathbf{A f}=l_{k, 1}, \quad \mathbf{n}=h_{k, 1}
$$

where now $h_{k, 1}$ is considered as noise and $l_{k, 1}$ the data we wish to recover. Here, the linear operator $\mathbf{A}$ is $\mathbf{A}=\mathbf{V} \boldsymbol{\Lambda}_{\epsilon}^{\infty} \mathbf{V}^{-1}$ and $\mathbf{f}=r_{k}$. 


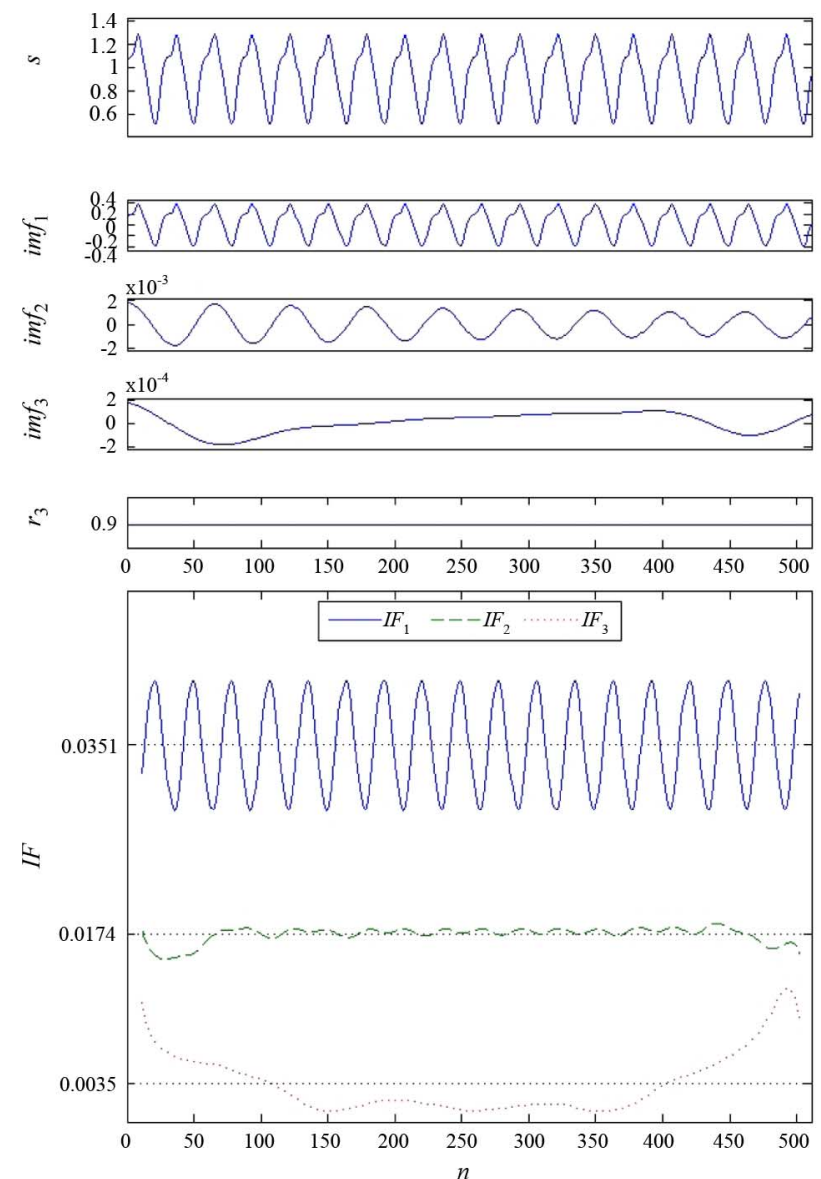

(a)
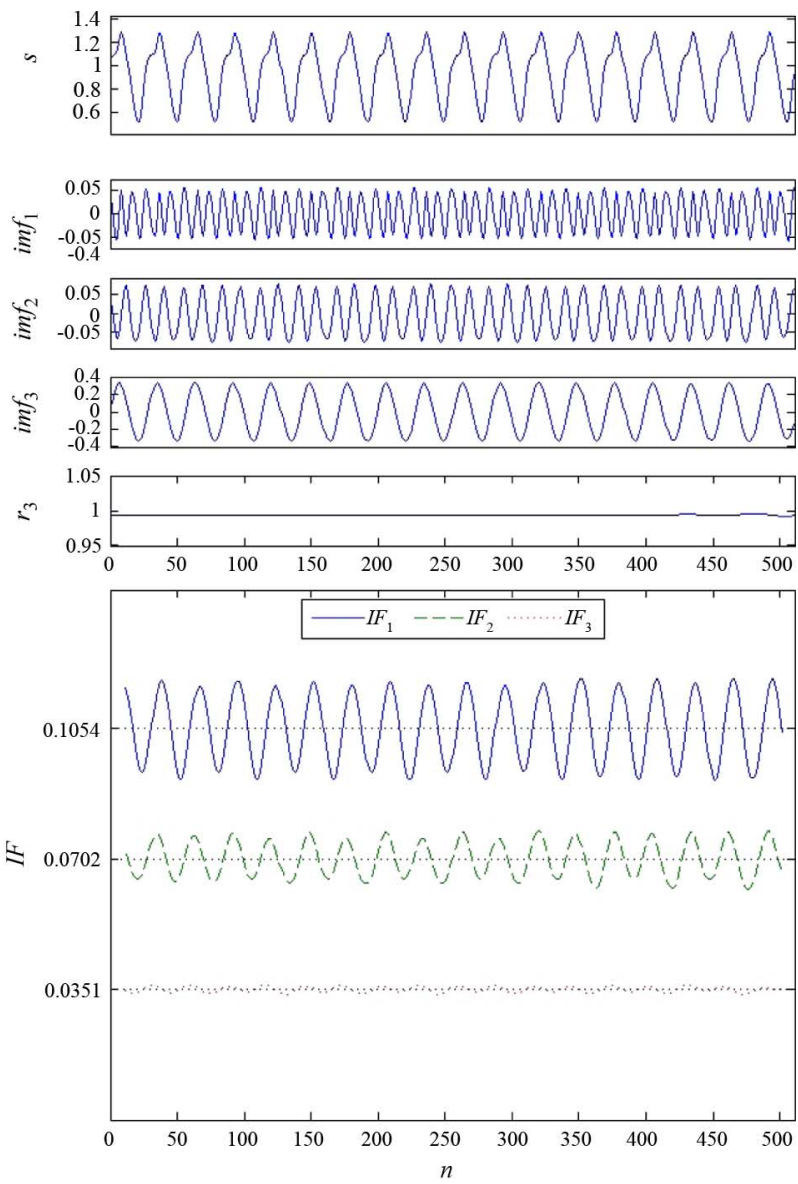

(b)

Fig. 7. EMD decomposition of a Mackey-Glass time series. (a)-(b) Top plot, the time series (18). Middle plots, IMFs and residual. Bottom plot, Instantaneous Frequency estimation for each IMF. (a) Decomposition result with original EMD algorithm. Note that the time series is essentially composed of the first IMF $i m f_{1}$. (b) Decomposition result with MCP-based approach resulting on three IMFs.

Therefore, we define the model error and the prior error as

$$
\begin{aligned}
& C_{\epsilon}\left(l_{k, 1}\right)=\left\|l_{k, 1}^{\epsilon}-r_{k}\right\|^{2} \\
& \Omega_{\epsilon}\left(l_{k, 1}\right)=\left\|\mathbf{L}_{2}\left(l_{k, 1}^{\epsilon}-l_{k, 1}\right)\right\|^{2}
\end{aligned}
$$

where $l_{k, 1}^{\epsilon}$ is the solution obtained from (17) for a fixed $\epsilon(0 \leq \epsilon \leq 1)$, and where the operator $\mathbf{L}_{2}$ is a matrix which approximates the second derivative. Here, $\epsilon$ acts as a regularization parameter such that $l_{k, 1}^{0}=l_{k, 1}$ and $l_{k, 1}^{1}=r_{k}$.

\section{Estimating Optimal Parameter Using Tikhonov Curve}

Perhaps the most convenient way for setting $\lambda$, in (20), is the "Tikhonov curve," or "L-curve" [17]. When we plot the prior error, $\log \left\|\mathbf{f}_{\lambda}-\mathbf{f}^{\infty}\right\|$, versus the model error, $\log \left\|\mathbf{d}-\mathbf{A} \mathbf{f}_{\lambda}\right\|$, we get the characteristic L-shaped curve with a (often) distinct corner separating vertical and horizontal parts of the curve, see Fig. 8. The optimal regularization parameter $\lambda$ is defined as that which strives to minimize and balance the two error terms. A tradeoff between these two error metrics is the use of regularization at the corner of the L-curve.

In the same way, the optimal $\epsilon$ is estimated from the L-curve obtained after plotting $\log \left\|l_{k, 1}^{\epsilon}-r_{k}\right\|$ versus $\log \left\|L_{2}\left(l_{k, 1}^{\epsilon}-l_{k, 1}\right)\right\|$ for $\epsilon \in[0,1]$. Here, $r_{k}$ is considered

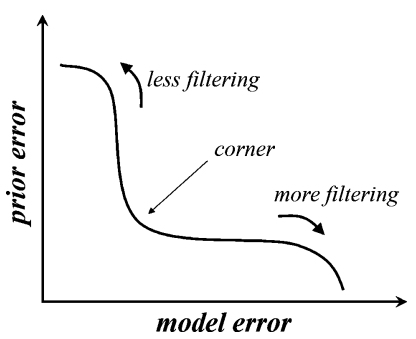

Fig. 8. The generic form of the L-curve. The curve is labeled parametrically with the value of the regularization parameter. The solution which appears optimal visually lies slightly to the neighborhood of the corner, e.g., the position of upwards-pointing curvature.

as the measured data, $l_{k, 1}$ denotes the prior information about the solution, and $l_{k, 1}^{\epsilon}$ is a regularized version of the solution.

\section{Numerical Experimentation}

To investigate the performance of the proposed approach we applied it to transient extraction, mode mixing separation, and to a "normal" composition. We noticed that the estimation of the optimal $\epsilon$ value can be carried out by applying the Tikhonov regularization to the proto-residual obtained after only one iteration of the sifting process. In order to locate the corners of the discrete L-curve, we use the algorithm proposed in [18]. Once 

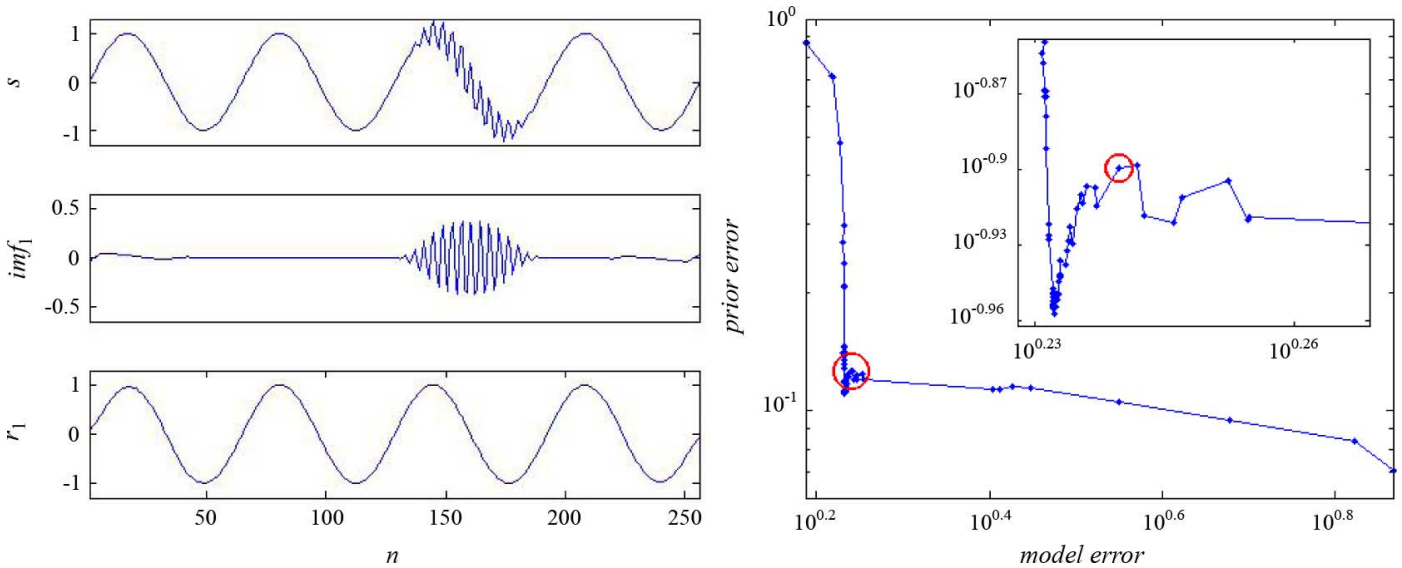

Fig. 9. Numerical experimentation for automatic regularization parameter estimation. A pure tone signal with a superimposed transient. The Tikhonov-curve is represented with detected corner marked with a circle, as well as the resulting decomposition using the estimated optimal parameter $\epsilon$.
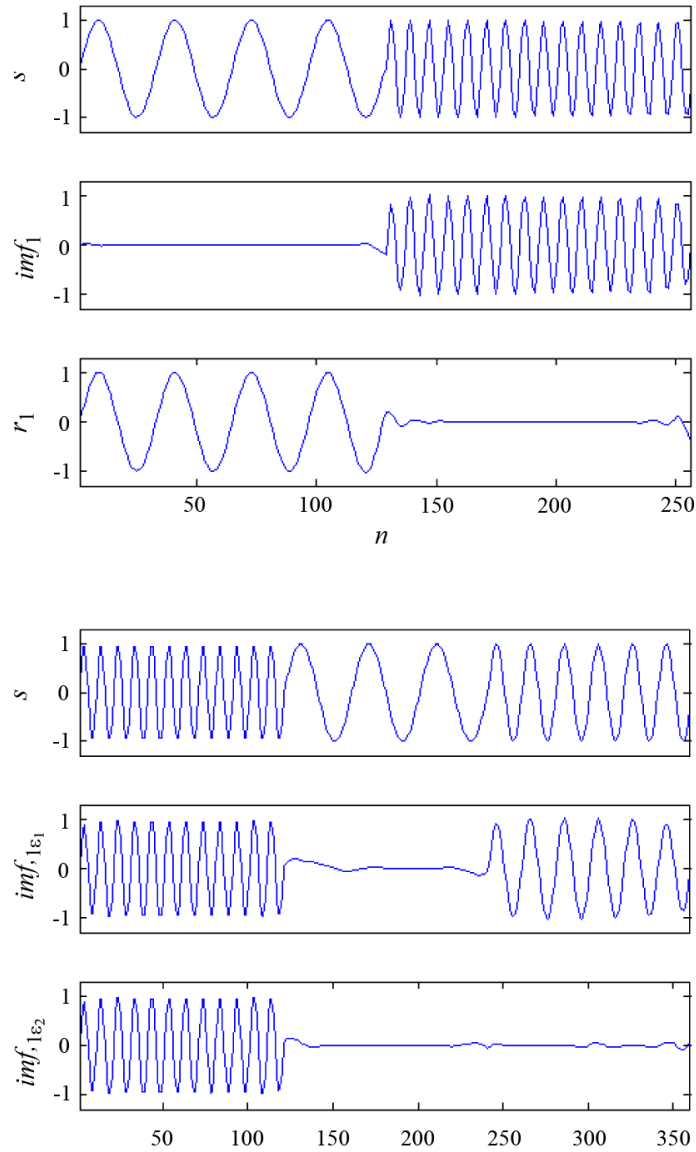

(b)

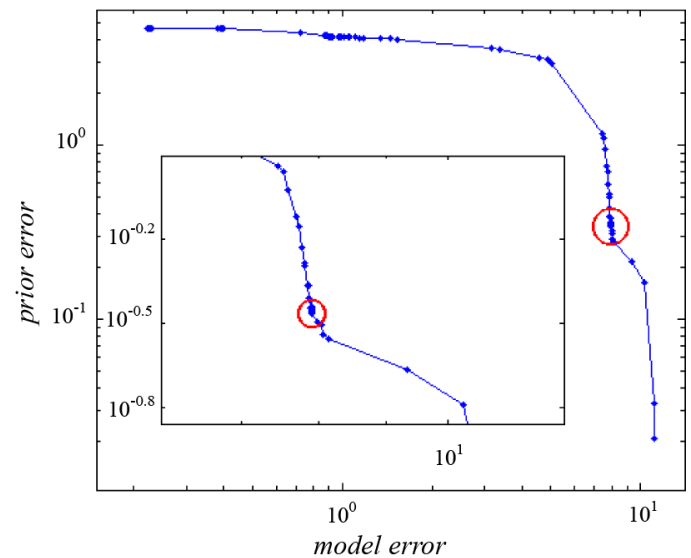

(a)

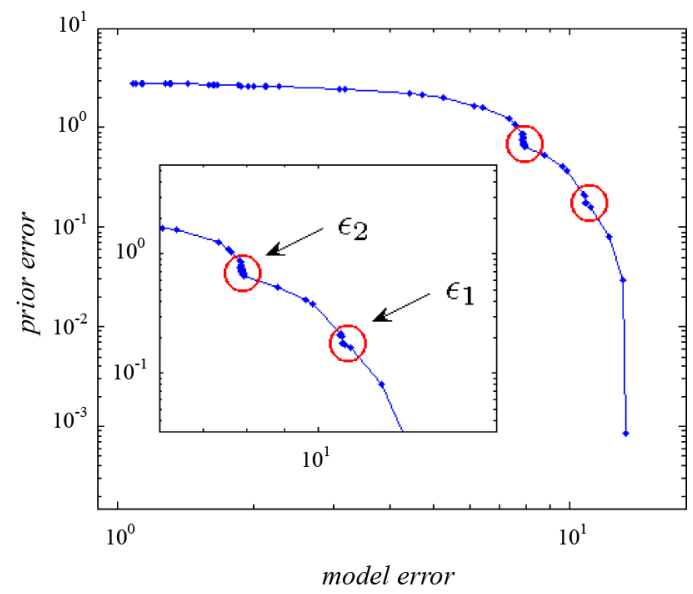

Fig. 10. Numerical experimentation for automatic regularization parameter estimation. (a) A mode mixing signal building with two successive pure tones. (b) A mode mixing signal building with three successive pure tones. In each case, the Tikhonov-curve is represented with detected corner marked with a circle, as well as the resulting decomposition using the estimated optimal parameter $\epsilon$.

that the first iteration is realized, the sifting process is carried out normally (with $\epsilon=0$ ) on porto-mode until obtaining the final IMF and residual.

1) Example 5 (Transient Extraction): The first example, reported in Fig. 9, concerns a transient superimposed to a pure tone signal. The associated L-curve exhibits a marked corner which is well detected. Hence, using the estimated optimal $\epsilon$ value, the first IMF, $i m f_{1}$, captures the transient, and the residual signal, $r_{1}$ is the transient-free pure tone.

2) Example 6 (2-Mode Mixing Separation in Time): The second example addresses the mode mixing separation problem. In this example, the signal is build by a concatenation of two pure tones with different frequencies, see Fig. 10(a). In this case, the curve exhibits a marked corner which is well detected. The 

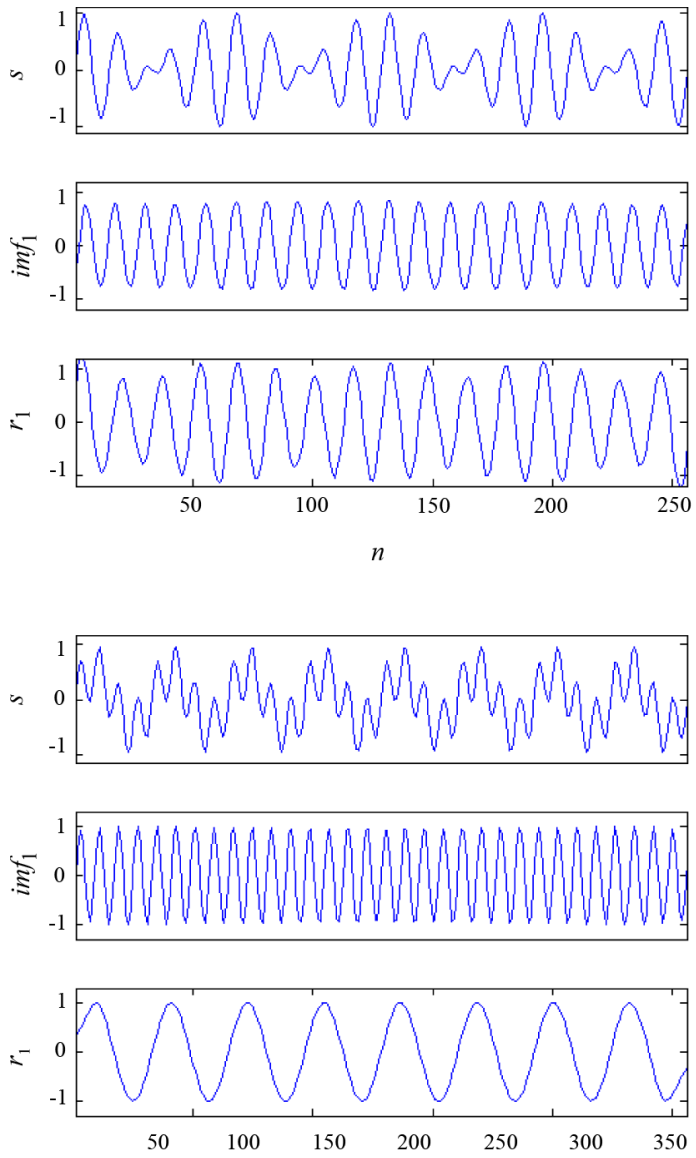

(b)

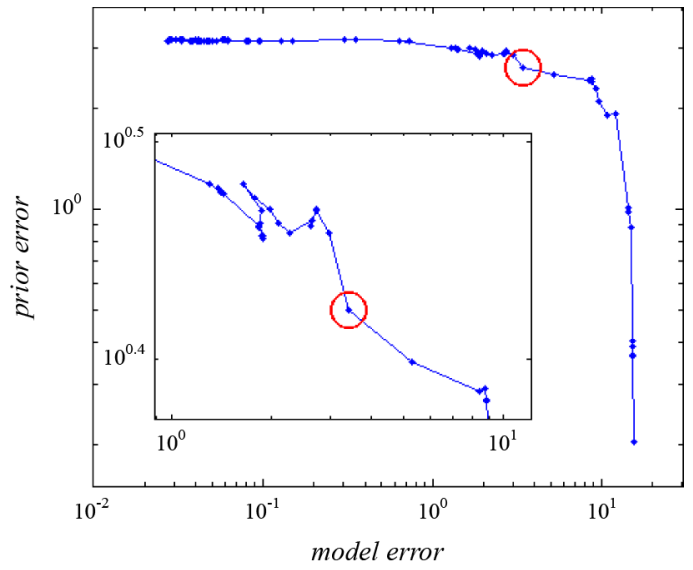

(a)

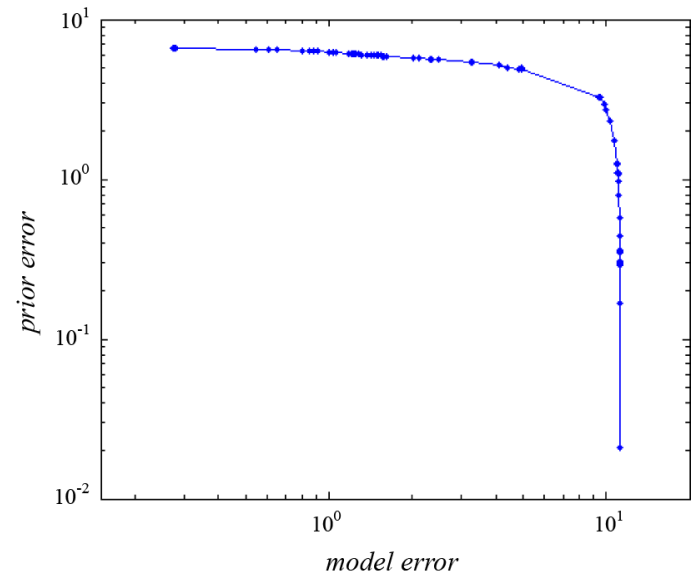

Fig. 11. Numerical experimentation for automatic regularization parameter estimation. (a) A mode mixing signal as a sum of two pure tones with close frequencies. (b) A "normal" case for which the signal is a sum of two pure tones with very different frequencies. In each case, the Tikhonov-curve is represented with detected corner marked with a circle, as well as the resulting decomposition using the estimated optimal parameter $\epsilon$.

use of the optimal $\epsilon$ value makes it possible to separate the two tones with the highest frequency one in $i m f_{1}$ and the lower in $r_{1}$.

3) Example 7 (3-Mode Mixing Separation in Time): The third example addresses the mode mixing separation problem. In this example, the signal is build by a concatenation of three pure tones with different frequencies, see Fig. 10(b). In this case, the curve exhibits two marked corners which are well detected. The use of the two optimal $\epsilon$ values, $\epsilon_{1}$ and $\epsilon_{2}$ makes it possible to separate the three tones as illustrated in Fig. 10(b).

4) Example 8 (2-Mode Mixing Separation in Frequency): This fourth example also addresses the mode mixing separation problem. In this example, the signal is sum of two pure tones with close frequencies, see Fig. 11(a). In this case, the curve exhibits a little marked corner but which is as well detected. The use of the optimal $\epsilon$ value makes it possible to separate the two tones with the highest frequency one in $i m f_{1}$ and the lower in $r_{1}$.

5) Example 9 (Normal Composition): Finally, the last example relates to a "normal" case for which the sifting process must be applied just as it is with $\epsilon=0$. Indeed, in this case the signal to analyze is the sum of two pure tones, see Fig. 11(b). One can note that the associated curve does not present a
L-shape as for the two preceding examples. So, no corner is detected and the optimal parameter is set to $\epsilon=0$, and the sifting process is performed to obtained the first tone in $i m f_{1}$ and the second one in $r_{1}$ as illustrated in Fig. 11(b).

\section{CONCLUSION}

It is a well-known fact that EMD method is developed on the basis of an algorithm and hence it suffers from a lack of a full and generally accepted theoretical framework. Thus, it is of great importance to develop an analytical formulation for the so called mean envelope to characterize this method. The main problem is due to the fact that the local mean of a signal depends on its characteristic local time-scales. In our previous paper, the utility of the PDE-based Framework method has been successfully demonstrated with the help of several synthetic signals, which shows that this approach performs as well as the classical EMD method. In this paper, a novel spectral approach, which estimates the mean-envelope of a signal has been presented. Here, we have extended the PDE-based approach in order to cover cases where classical EMD is quite weak for signal analysis. It is the case of signals without local extrema, or chaotic signals like chaotic time series. This extension is on one hand based on MCP 
of a signal and on another hand on a regularization approach inspired by the well known Tikhonov regularization method. So, we have also proposed a quasi-asymptotic solution, to deal with transient component or mode mixing signals. We have shown how it can resolve automatically the mode mixing and transient problems, and have proven its powerfulness in the case of complex signals like chaotic time series. The formulation proposed here is believed to provide with new insights in EMD and calls for further studies. In our future works, this spectral approach will be used on real signals for purpose of analysis and characterization of physical data.

\section{REFERENCES}

[1] N. E. Huang, Z. Shen, S. R. Long, M. L. Wu, H. H. Shih, Q. Zheng, N. C. Yen, C. C. Tung, and H. H. Liu, "The empirical mode decomposition and Hilbert spectrum for nonlinear and non-stationary time series analysis," in Proc. R. Soc. London A, 1998, vol. 545, pp. 903-995.

[2] G. Rilling, P. Flandrin, and P. Gonçalvès, "On empirical mode decomposition and its algorithms," in IEEE-EURASIP Workshop on Nonlin. Signal Image Process. , NSIP-03, Grado (I), 2003.

[3] Q. Chen, N. Huang, S. Riemenschneider, and Y. Xu, "A B-spline approach for empirical mode decompositions," Adv. Comp. Math., vol. 24, no. 1, pp. 171-195, 2006.

[4] R. Sharpley and V. Vatchev, "Analysis of the intrinsic mode functions," Construct. Approx., 2005.

[5] E. Deléchelle, J. Lemoine, and O. Niang, "Empirical mode decomposition: An analytical approach for sifting process," IEEE Signal Process. Lett., vol. 12, pp. 764-767, 2005.

[6] O. Niang, "Empirical mode decomposition: contribution à la modélisation mathématique et application en traitement du signal et l'image," Ph.D., Univ. Paris 12, Créteil, Paris, France, 2007.

[7] [Online]. Available: http://perso.ens-lyon.fr/patrick.flandrin/emd.html

[8] P. Flandrin, G. Rilling, and P. Gonçalvès, "Empirical mode decomposition as a filter bank," IEEE Signal Process. Lett., vol. 11, pp. 112-114, 2004.

[9] Z. Wu and N. E. Huang, "A study of the characteristics of white noise using the empirical mode decomposition method," Proc. R. Soc. London A, vol. 460, no. 2046, pp. 1597-1611, 2004.

[10] N. E. Huang, M. Wu, S. Long, S. Shen, W. Qu, P. Gloerson, and K. Fan, "A confidence limit for the empirical mode decomposition and Hilbert spectral analysis," Proc. R. Soc. London A, vol. 459, no. 2037, pp. 2317-2345, 2003.

[11] N. E. Huang, Z. Shen, and S. R. Long, "A new view of nonlinear water waves: The Hilbert spectrum," Ann. Rev. Fluid Mechan., vol. 31, pp. 417-457, 1999.

[12] Z. Wu and N. E. Huang, "Ensemble empirical mode decomposition: A noise assisted data analysis method," Adv. Adapt. Data Anal., vol. 1, no. 1, pp. 1-41, 2009.

[13] R. Deering and J. F. Kaiser, "The use of a masking signal to improve empirical mode decomposition," in Proc. ICAASP, 2005, pp. 485-488.
[14] J. D. Murray, "Mathematical biology," in Biomath. Texts, 2nd ed. New York: Springer-Verlag, 1993, vol. 19.

[15] M. C. Mackey and L. Glass, "Oscillation and chaos in physiological control systems," Science, vol. 177, pp. 287-289, 1977.

[16] A. N. Tikhonov and V. I. Arsenin, Solutions of Ill-Posed Problems. Washington/New York: Halsted, 1977.

[17] P. C. Hansen and D. P. O'Leary, "The use of the L-curve in the regularization of discrete ill-posed problems," SIAM J. Sci. Comput., vol. 14, pp. 1487-1503, 1993.

[18] G. Rodriguez and D. Theis, "An algorithm for estimating the optimal regularization parameter by the L-curve," Rendiconti di Matematica, Serie VII, vol. 25, pp. 69-84, 2005.

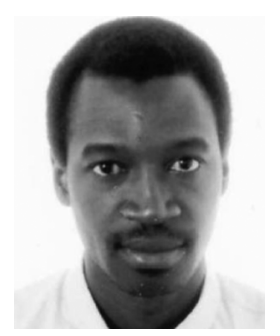

Oumar Niang received the Ph.D. degree in computer sciences from LISSI-E.A. 3956, Univ-Paris-Est Créteil Val-de-Marne, in 2007.

$\mathrm{He}$ is a Research Professor with the Ecole Polytechnique de Thies Sénégal. He is a member of LANI, UGB Sénégal, with research interests focused on mathematical modeling in signal and images processing, time frequency analysis, biomedical signal, and medical image analysis.

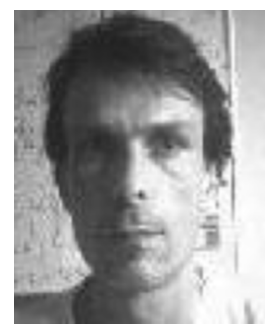

Éric Deléchelle received the Ph.D. degree in biomedical engineering from the Université Paris Est Créteil Val-de-Marnel, France, in 1997.

$\mathrm{He}$ is with the Laboratoire Images, Signaux et Systèmes Intelligents (LiSSi-EA 3956), Université Paris 12 Val-de-Marne, Créteil. Since 1999, he has been Maitre de Conférences at the Institut Universitaire de Technologie, Créteil, with research interests focused on stochastic signal analysis, biomedical signal, and medical image processing.

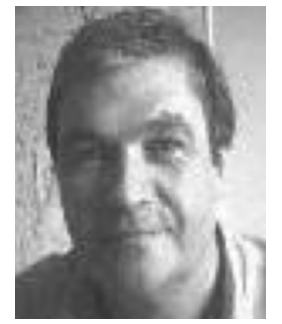

Jacques Lemoine received the Ph.D. degree in biomedical engineering from the Université Paris Est Créteil Val-de-Marnel, France, in 1981.

$\mathrm{He}$ is a Professor in the Laboratoire Images, Signaux et Systèmes Intelligents (LiSSi-EA 3956), UFR des Sciences et Technologie, Université Paris Est Créteil Val-de-Marne, France. His research interests are focused on stochastic signal analysis, biomedical signal, and medical image processing. 